\title{
APLICACIÓN DEL E-COMMERCE PARA EMPRENDIMIENTOS ECUATORIANOS EN TIEMPOS DE DISRUPCIÓN
}

\section{Application of E-Commerce for Ecuadorian Businesses in times of Disruption}

\author{
Jhonson Orlando Cando Cedeño, Est. \\ Instituto Tecnológico de Formación Profesional \\ Administrativa y Comercial, Ecuador \\ https://orcid.org/0000-0003-4035-6135 \\ jocando@formacion.edu.ec
}

\author{
Jordan Lindon Cando Cedeño, Ing. \\ Universidad de Guayaquil, Ecuador \\ https://orcid.org/0000-0001-6375-3972 \\ jordan.candoc@ug.edu.ec
}

Palabras claves: Millenials, Starups, E-Commerce, Mypimes, Emprendimiento.

Keywords: Millenials, Starups, E-Commerce, Mypimes, Entrepreneurship.
Recibido: 07 de enero de 2021

Aceptado: 24 de marzo de 2021

\section{RESUMEN}

Ecuador es un país emprendedor, ya lo detallan así, los indicadores globales de emprendimiento a nivel joven y es un referente latinoamericano en esta área, por lo tanto, debido a estos antecedentes se efectúa el análisis del potencial emprendedor que tendría la nación al apostar por las plataformas digitales, marcando las tendencias tecnológicas actuales optadas por los millenials, y diversas generaciones, que van de la mano del comercio electrónico, ya que en la ideología moderna si no está en línea no existe y es así como muchos negocios se ven en la necesidad de obtener presencia digital para no decaer en estos tiempos de disrupción, se propone como metodología recurrir a la revisión documental de indicadores a nivel país, regional y mundial; lo cual supone una mejor apreciación de la realidad ecuatoriana en contraste con otras naciones, como unos de los resultados más importantes se prevé una mejora en el actual situación de starups, emprendimientos y mypimes recientes y ya consolidados.

\begin{abstract}
Ecuador is an entrepreneurial country, as detailed by the global indicators of entrepreneurship at a young level and is a Latin American reference in this area, therefore, due to this background the analysis of the entrepreneurial potential that the nation would have when betting on the digital platforms, marking the current technological trends millennials, and various generations, go hand in hand with electronic commerce, since in modern ideology if it is not online it does not exist and that is how many businesses find themselves in need of presence digital not to decay in these times of disruption, it is proposed as a methodology to resort to the documentary review of indicators at the country, regional and global levels; This implies a better appreciation of the Ecuadorian reality in contrast to other nations, as one of the most important results is an improvement in the current situation of starups, ventures and recent and consolidated mypimes.
\end{abstract}




\section{INTRODUCCIÓN}

Internet ha revolucionado cada aspecto de nuestras vidas. No solo ha cambiado la manera en que se comparte la información, sino también en cómo llevamos a cabo nuestras actividades cotidianas y satisfacemos nuestras necesidades más básicas, comida y ropa. La era digital ha transformado nuestras vidas: inicialmente, se compraba comida en un mercado, quizá en una tienda local o en un supermercado, pero ahora se pide en línea para que sea entregada en nuestros hogares. (Sigmond, 2018)

Hasta el momento, solo los gigantes tecnológicos y las empresas que son nativas digitales han podido responder con éxito a la transformación digital, explotando la tecnología y desarrollando nuevos modelos de negocio basados en plataformas digitales. Este modelo les ha permitido dominar en términos de crecimiento, beneficio y capitalización bursátil, creando al mismo tiempo sus propios ecosistemas digitales. Sin embargo, las empresas tradicionales también tienen la oportunidad de convertirse en disruptores si transforman sus modelos de negocio y se benefician del nuevo entorno digital. Necesitan aplicar los exitosos modelos de plataforma digital para crear entornos comunes entre sus socios, proveedores y consumidores en donde puedan ofrecer más valor y servicios añadidos con ayuda de terceros. (Zamora, 2016)

Es por lo antes mencionado que los jóvenes emprendedores ecuatorianos, deben convertirse en los disruptores de este panorama digital y enfocarse en los negocios que permitan generar ventas a través de internet, pero no sólo los millenials pueden acogerse a este modelo de negocio como lo explica el autor Zamora, las empresas tradicionales también adaptar sus actividades a este nuevo modelo, y de esa forma poder contar tanto con presencia on-line como off-line.

Actualmente, el nivel de empleabilidad de una persona se incrementa con el desarrollo de las llamadas habilidades "blandas", entre las que destacan: trabajo en equipo, multidisciplinariedad, disposición al aprendizaje continuo y capacidad de emprendimiento [...] el espíritu de liderazgo, las habilidades organizativas y la propensión al riesgo tienen un efecto positivo sobre la intención emprendedora de los jóvenes. (Montalvo, 2016)

Para poder ser un emprendedor no sólo basta con la convicción y la generación de una idea exitosa, también es necesario tener ciertas habilidades que le permitan poder mantenerse y adaptar su modelo de negocio al mercado debido a que este es muy cambiante y así mantener al margen a la competencia. A pesar de que se puede emprender en solitario, es un trabajo muy arduo y según algunos expertos es mejor emprender en asociatividad, de esta forma se pueden equiparar las habilidades de cada uno de los participantes del starup, creando valor para la idea de negocio puesto que las falencias de un miembro del equipo pueden ser reforzadas por las habilidades de otro, obteniendo un aprendizaje continuo.

Según (Ortiz, Camacho, \& Beltrán, 2017) el comercio electrónico a nivel mundial se ha incrementado considerablemente en los últimos 10 años, grandes empresas de comercio al por menor con presencia física, están considerando como alternativa el comercio electrónico; las empresas y consumidores ecuatorianos comienzan a utilizar el comercio electrónico como un nuevo canal de compras/ventas, en el país es incipiente este tipo de comercio con respecto a países como Brasil, México y Argentina al tenerse un número limitado de empresas que han incursionado en este tipo de comercio y el bajo acceso que tiene la población al servicio de internet.

En relación a lo mencionado por los anteriores autores, es que se realiza la siguiente hipótesis si se mejoran las falencias en el modelo de e-commerce ecuatoriano entonces aumentaran los emprendimientos digitales, esto puede ser factible debido a que más adelante se abordaran los aspectos negativos que ha tenido el comercio electrónico en nuestro país y se presentará una perspectiva de cómo se puede mejorar e incursionar en este ámbito.

Es necesario animar al pequeño empresario a lanzase al comercio electrónico, ya que una empresa pequeña tiene posibilidades de triunfar en el e-commerce, debido a que al ser más pequeña su estructura es más ágil y esto le permite adaptarse mejor a nuevas formas de trabajar. Respecto a la influencia en la capacidad de innovación, el factor "edad" influye de manera notoria, por lo que cuanto más tiempo lleva la empresa en el mercado, esta tiene más relaciones y experiencia. (Fernández, Sánchez, Jiménez, \& Hernández, 2015)

La adopción del comercio electrónico en las empresas requiere de acceso físico o conectividad a Internet; factores relacionados con las capacidades internas de cada organización; y condiciones políticas, sociales y económicas del contexto que varían de una mipyme a otra. En todo caso, la empresa debe aprender gradualmente a utilizar las TIC, realizando esfuerzos internos para desarrollar las competencias organizacionales específicas que requieren el uso y la aplicación de TIC en procesos de gestión y comercialización. (Sanabria, Torres, \& López, 2016) 
Con respecto al tema de seguridad al llevar a cabo actos de comercio a través de internet, es importante señalar que nuestro país aún se encuentra lejos de contar con las mejores condiciones. Es por esto que se deben crear campañas de información sobre el comercio electrónico poniendo especial atención a medidas que permitan evitar ser víctima de algún delito como el robo de datos personales o fraude. (Ríos, 2015)

\section{DESARROLLO}

La metodología aplicada en este trabajo de investigación es de tipo secundaria ya que se recurrió a informes, documentación bibliográfica y demás fuentes que no están relacionados directamente con el fenómeno de estudio, a su vez la investigación no fue de campo si no de forma documental, es debido a esto que se realizó tablas y gráficos para establecer comparativas entre los factores que inciden en el e-commerce, además se recurrió a encuestas realizadas sobre esta temática para contextualizar la realidad ecuatoriana sobre los puntos a mejorar en el comercio electrónico ecuatoriano.

\section{ÍNDICES DE EMPRENDIMIENTO ECUATORIANO}

En la tabla. 1 podemos observar que a nivel de región ecuador se encuentra por debajo de la media en cuanto a las ventas anuales de comercio electrónico con un valor en promedio de $\$ 800,000,000$ con lo que cerró el año 2018 pero a su vez posee un gran potencial en el porcentaje de penetración de Internet con $80 \%$, esto quiere decir que tiene la posibilidad de alcanzar y superar a sus competidores directos, cabe recalcar que la actividad del e-commerce no está del todo desarrollada en nuestro territorio en comparación con las grandes potencias mundiales, pero el desconocimiento y la inseguridad informática que sienten los compradores locales es muy alta, por ende son puntos a considerar al momento de incursionar en este ámbito tecnológico.

Tabla 1. índices de comercio electrónico en América

\begin{tabular}{lrr}
\hline País & $\begin{array}{c}\text { Las ventas anuales de } \\
\text { comercio electrónico }\end{array}$ & $\begin{array}{c}\text { El porcentaje de } \\
\text { penetración de } \\
\text { Internet }\end{array}$ \\
\hline EUA & $\$ 504,582,000,000$ & $88 \%$ \\
México & $\$ 17,629,609,834$ & $65 \%$ \\
Canadá & $\$ 39,966,000,000$ & $90 \%$ \\
Brasil & $\$ 19,722,580,000$ & $71 \%$ \\
Chile & $\$ 5,888,000,000$ & $78 \%$ \\
Argentina & $\$ 4,260,738,000$ & $93 \%$ \\
Colombia & $\$ 4,000,000,000$ & $63 \%$ \\
Perú & $\$ 4,000,000,000$ & $68 \%$ \\
Ecuador & $\$ 800,000,000$ & $80 \%$ \\
Venezuela & $\$ 449,000,000$ & $53 \%$ \\
\hline
\end{tabular}

Como se mencionó anteriormente ecuador es un país con un alto índice de TEA o Actividad Emprendedora Temprana, así lo expresa el estudio efectuado por el GEM en el 2017, marcando a Ecuador como un referente regional americano en emprendimiento, es decir los habitantes de este país tienen un alta predisposición a efectuar negocios y generar sus propios negocios, muchas veces esto está dado por la necesidad de laboral dada la escasez de trabajo, o en el caso de empleados que buscan aumentar su economía con un ingreso adicional a razón de un emprendimiento ya sea en solitario o a través de la colaboración con otros.

Gráfico 1. Índices de Actividad Emprendedora Temprana (TEA) 


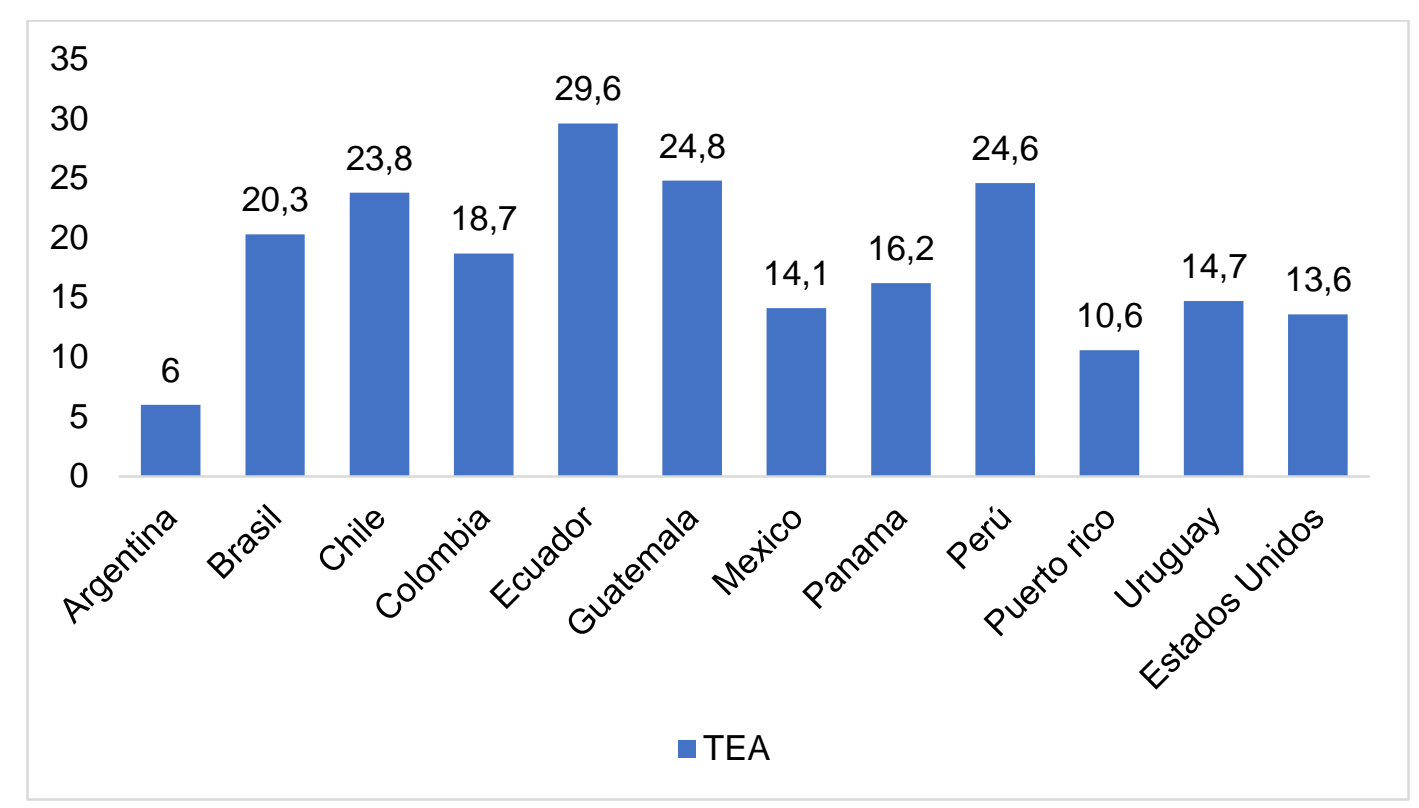

Fuente: GEM, 2017

Elaborado por: Los Autores

En el gráfico 3 puede apreciarse el Esquema del Ecuador según el Índice Global de Emprendimiento (GEDI), el Monitor Global de emprendimiento (GEM), responsable de dicho análisis, se basa en 14 pilares fundamentales para tener una idea más clara sobre el ecosistema emprendedor de cada país, es así que en Ecuador un punto a destacar son las habilidades de inicio de sus emprendimientos es decir que existe una alta iniciativa para poder emprender en el país, en el caso de las redes como ya se evidenció anteriormente no es tan alto pero sin embargo es bastante aceptable en consideración de la media regional, por ende existe un océano azul, el cual no ha sido explotado en su totalidad y una oportunidad para que los nuevos emprendimientos ecuatorianos, opten por aplicar sus recursos en las redes, propiamente en el comercio electrónico o e-commerce.

\section{Gráfico 2. Esquema del Ecuador según el Índice Global de Emprendimiento}

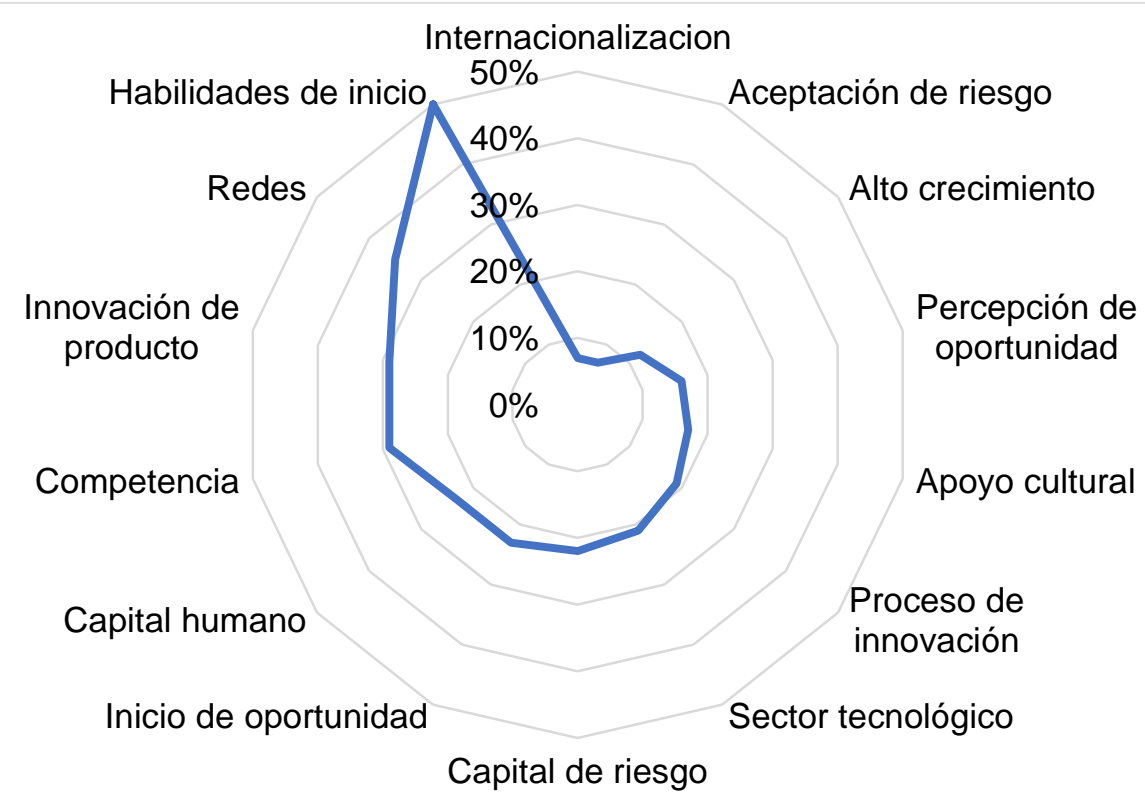

Fuente: GEDI, 2018

Elaborado por: Los Autores 


\section{TENDENCIAS LOCALES Y MUNDIALES DEL E-COMMERCE}

Respecto a las tendencias de compras a nivel mundial de forma digital estas se caracterizan por en una gran cantidad por libros, música películas y video juegos así lo demuestra el grafico 3 con un $60 \%$, en cuanto a las compras en físico a través de las tiendas tradicionales el mayor consumo está dado por las tiendas de comestibles en un $70 \%$, puesto que los clientes prefieren poder ver y palpar lo que van a consumir, reduciendo de esta forma los alimentos maltratados o en mal estado, que pueden estropearse en el transcurso del envió, o en el caso de los alimentos perecibles, que es mejor consumirlos frescos, aunque también se da el caso de los alimentos importados pero ese es un tema aparte.

Gráfico 3. Tendencias mundiales de compras por internet

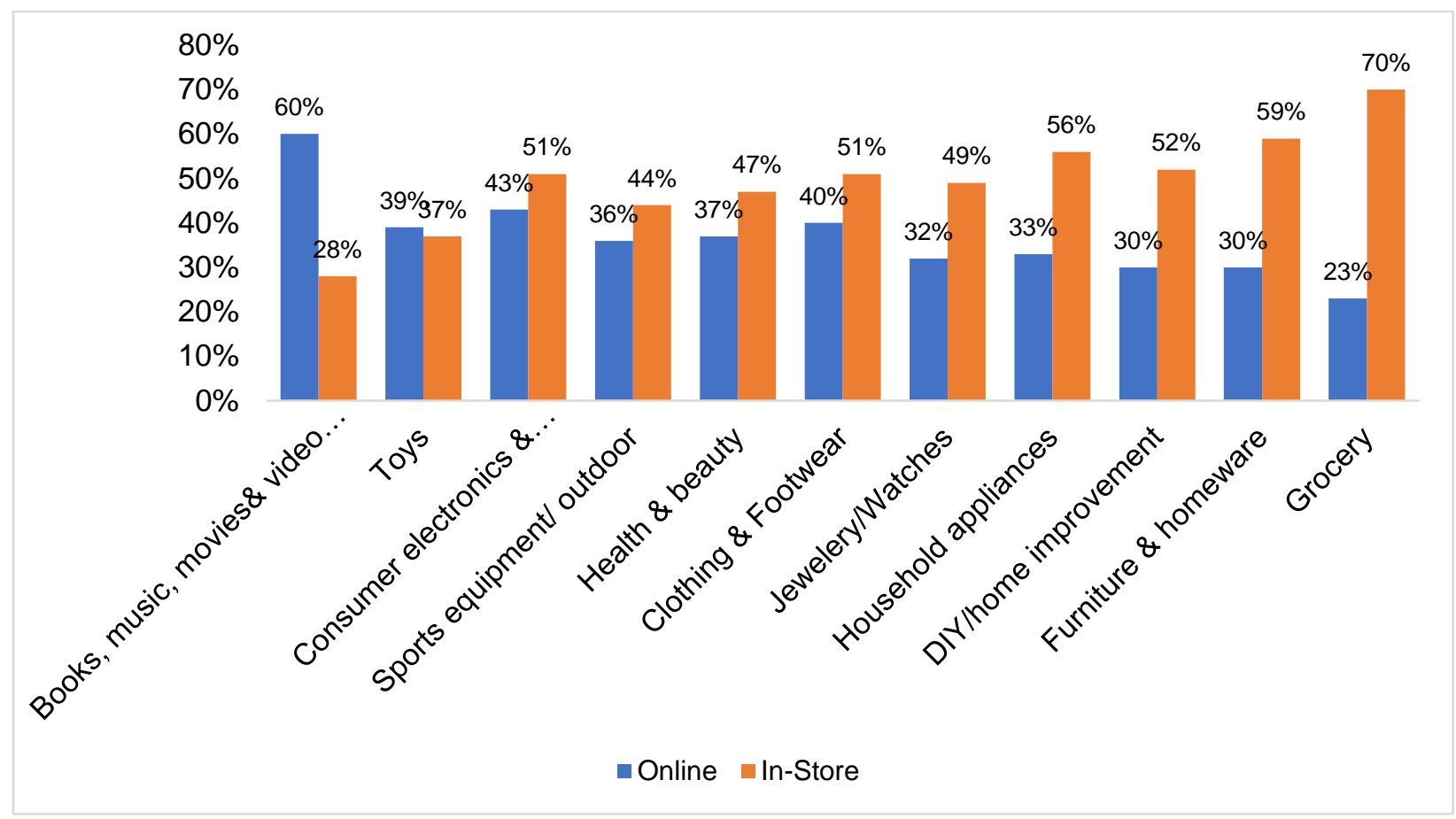

Fuente: Statista, 2018

Elaborado por: Los Autores

Por otro lado en cuanto a las tendencias de compras a través de internet en el país se registra según el Inec que, un $33 \%$ de las personas empleadas en dicho estudio prefieren adquirir prendas de vestir y calzado, y en un porcentaje casi similar un $31 \%$ prefieren comprar bienes y servicios diversos, como artículos de primera necesidad y de uso personal, esto enfoca más hacia la realidad ecuatoriana y permite direccionar los esfuerzos de los nuevos emprendimientos a invertir sus recursos en estos aspectos ya que van a tener una mayor acogida al adentrarse en dichos nichos de mercado.

Gráfico 4. Tendencias nacionales de compras por internet 


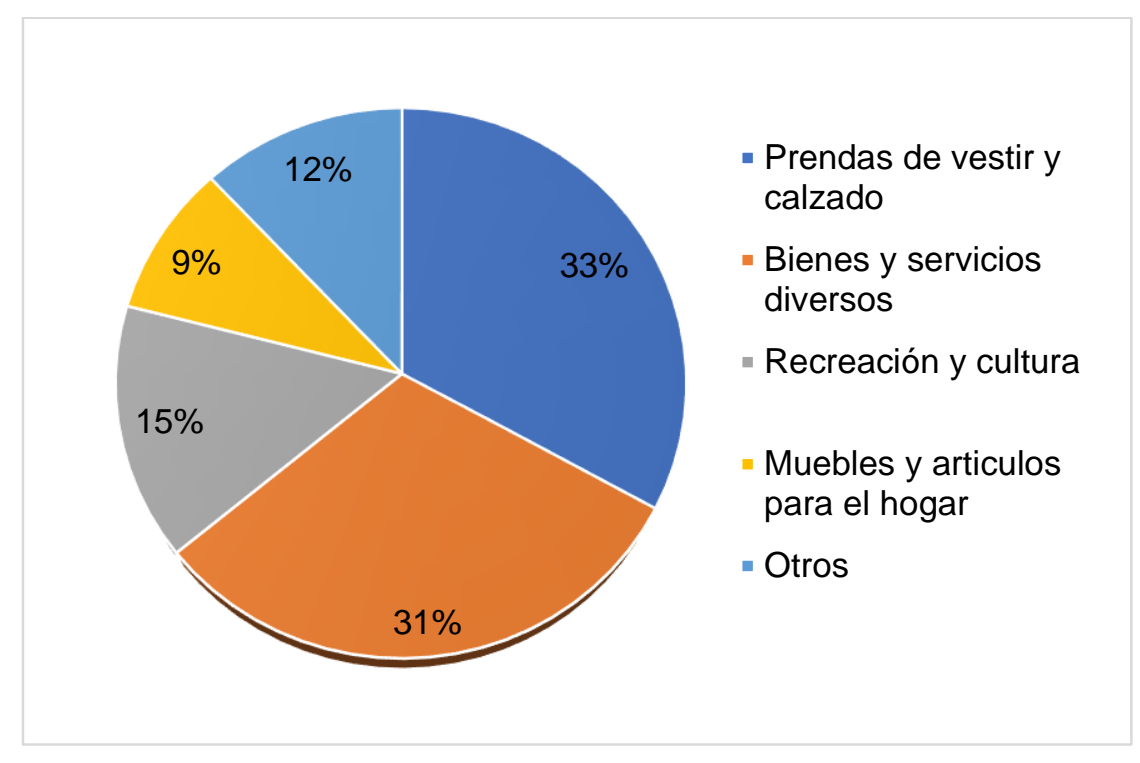

Fuente: Inec, 2015

Elaborado por: Los Autores

\section{CRECIMIENTO DEL E-COMMERCE EN EL ECUADOR}

El comercio electrónico en el Ecuador a pesar de no tener una maduración tan influyente como en países de Norteamérica o en Europa ha mantenido un crecimiento bastante estable a lo largo de la última década, por lo cual es un mercado bastante optimo en el cual pueden incursionar los jóvenes emprendedores del Ecuador, e inclusive las Pymes que ya cuentan con una infraestructura física incorporada, es decir se busca aprovechar los momentos de disrupción o de cambio de las nueva y emergente tendencia digital para la generación de más ingresos, empleos y mejorar la economía del país.

Tabla 2. Transacciones comercio electrónico Ecuador (USD)

\begin{tabular}{cr}
\hline Año & USD \\
\hline 2010 & $\$ 280.000 .000$ \\
2011 & $\$ 300.000 .000$ \\
2012 & $\$ 300.000 .000$ \\
2013 & $\$ 800.000 .000$ \\
2014 & $\$ 540.000 .000$ \\
2015 & $\$ 800.000 .000$ \\
2016 & $\$ 850.000 .000$ \\
2017 & $\$ 800.000 .000$ \\
2018 & $\$ 1.000 .000 .000$ \\
\hline Fuente: SeoQuito, 2016 \\
Elaborado por: Los Autores
\end{tabular}

Por otro lado, según (Korntheuer, 2016), por los impuestos y salvaguardias altas en el Ecuador esta forma de hacer compras online ha perdido un poco de popularidad. Las impuestos y salvaguardias ayudan al crecimiento del comercio electrónico local (si no vende productos importados). Sin embargo, mucha gente en el país realiza compras online en los EEUU a través de amigos a familiares que viven allá Luego, cuando ellos visitan Ecuador pueden traer los productos. 


\section{Gráfico 5. Crecimiento de las Transacciones comercio electrónico Ecuador (USD)}

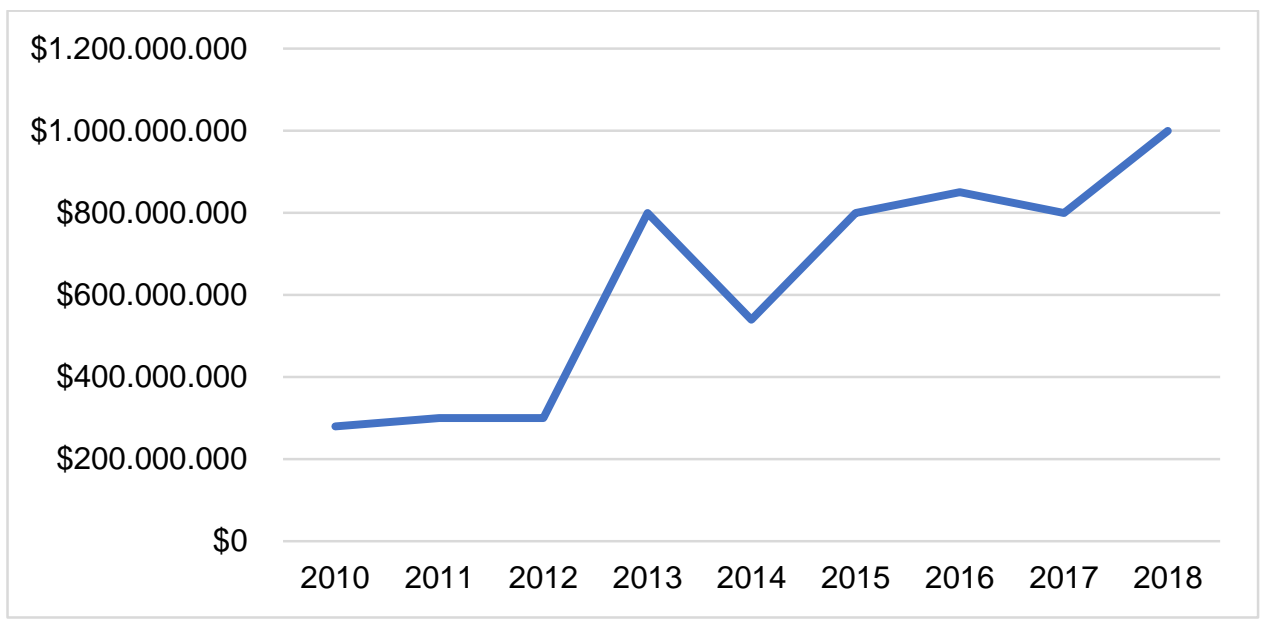

Fuente: SeoQuito, 2016

Elaborado por: Los Autores

\section{DESVENTAJAS DEL ECOMMERCE EN EL ECUADOR}

Según (Pino \& Salazar, 2018) la tecnología ayuda a la habilidad de consumo en ventajas como el hecho de que no hay que hacer colas para comprar, acceso a tiendas y productos en lugares remotos, es posible encontrar una gran cantidad de opciones, las tiendas online están disponibles todos los días a todas las horas, transacciones y contrataciones rápidas y eficientes, etc. entre las desventaja del cliente, tenemos la falta de comunicación y relación personal, imposibilidad de probar el producto antes de comprarlo, miedo a los pagos fraudulentos, a las estafas y al robo de la información personal (hackers), dificultad o incluso incapacidad para detectar las estafas y a los estafadores, incomodidad para las devoluciones, retraso en la recepción de los productos, etc.

El comercio electrónico está creciendo. Hay cada vez más internautas, especialmente a través de teléfonos inteligentes en el país. Al mismo tiempo, impuestos para productos importados hacen las compras dentro del Ecuador más atractivas. Sin embargo, hay algunos obstáculos que impiden que el comercio electrónico aumente de forma más rápidamente. Estos obstáculos son por ejemplo el miedo a hacer compras en línea o los problemas de implementar formas de pago como el pago a través de tarjetas de crédito en las tiendas online. (Korntheuer, 2016)

En el ecuador una gran parte de los usuarios indican tener miedo a fraudes o a engaños al momento de realizar compras por internet, seguido de una gran desconfianza en el pago, ya que no están tan familiarizados con la tecnología y en ocasiones nuevos emprendimientos con marcas no tan conocidas o populares caen en la desconfianza e inseguridad de los posibles compradores los cuales, se hallan más a gusto con marcas de renombre o las cuales puedan tener una tienda física a la cual acudir en caso de algún tipo de reclamo-

\section{Gráfico 6. Razones para no comprar en Línea}






Fuente: CECE - UESS, 2018

Elaborado por: Los Autores

Los motivos para no comprar en línea que se detallan en el gráfico 6 y en el gráfico 7 donde se indican los principales motivos para que se dé el abandono de una compra son de vital importancia, puesto que una página web o un medio digital puede poseer un alto tráfico de usuarios, pero si estos no llegan a concretar una compra no se está cumpliendo la razón de ser del e-commerce y por ende estos negocios digitales llegan a fracasar.

Según el estudio efectuado por la UESS, el principal indicador en los usuarios ecuatorianos son los problemas con la pagina o aplicación en un $52 \%$, seguido de las dudas en un $39 \%$, por lo que los puntos a mejorar deben ser la portabilidad de la página con los diferentes medios tecnológicos y contar con una interfaz más amigable con el usuario, además de poder reducir esa duda que poseen los usuarios a través de un manejo más eficaz del marketing que brinde una mayor seguridad del producto o servicio.

\section{Gráfico 7. Abandono de compra}

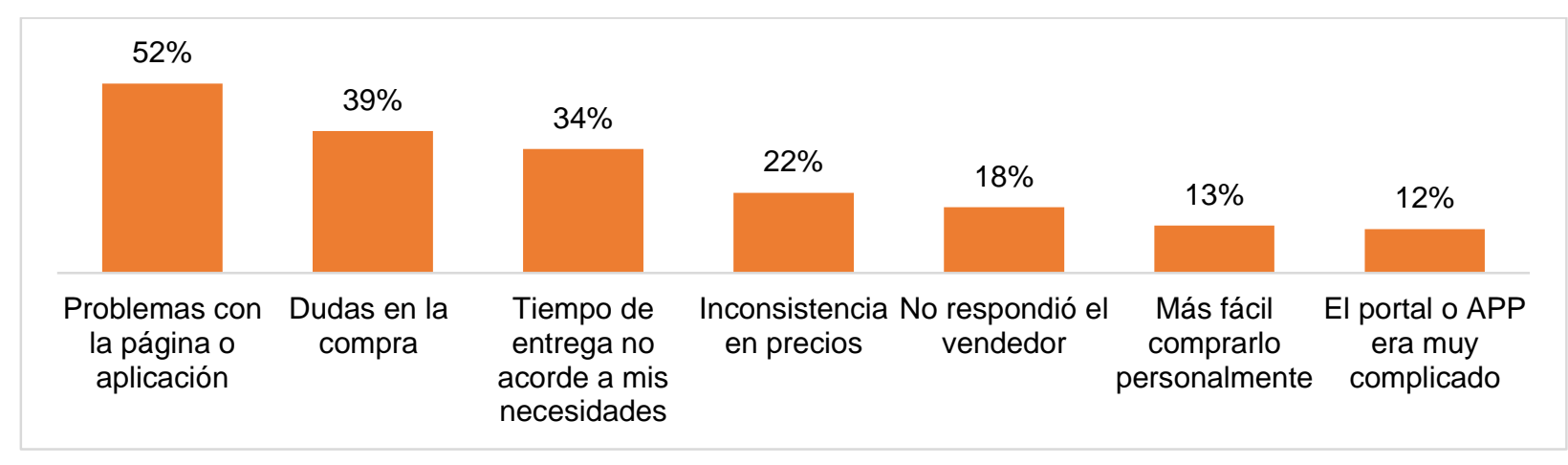

Fuente: CECE - UESS, 2018

Elaborado por: Los Autores

\section{MEJORAS EN EL E-COMMERCE ECUATORIANO A TRAVÉS DEL MARKETING DIGITAL}

En el Ecuador existen tiendas únicamente online, marketplaces, o personas informales que venden sus productos usándolas redes sociales, sin mayores controles de seguridad; [...] lo que caracteriza a la revolución tecnológica no es el carácter central del conocimiento y la información, sino la aplicación de ese conocimiento e información a aparatos de generación de conocimiento y procesamiento de la información o comunicación, en un cırculo de retroalimentación acumulativo entre la innovación y sus usos. (Esparza, 2017)

En el siguiente gráfico observamos un estudio efectuado el Inec a 28.846 viviendas distribuidas en 2.425 sectores del país, obteniendo que mayormente los usuarios ecuatorianos emplean la red social Facebook de la siguiente manera: a través del Smartphone en un $85,20 \%$; en la Tablet en un 84,30 y sus PC's o laptops en un $66,40 \%$; estos datos son de vital importancia puesto que aquí se identifica en que medios se pueden enfocar el marketing para aumentar el 
interés de los usuarios y concretar el mayor número de ventas posible, además de tener en cuenta la mejora continua de las plataformas que utilicen para que estén más adecuadas al uso de Smartphone's.

Gráfico 8. Filiación a redes sociales según dispositivo usado

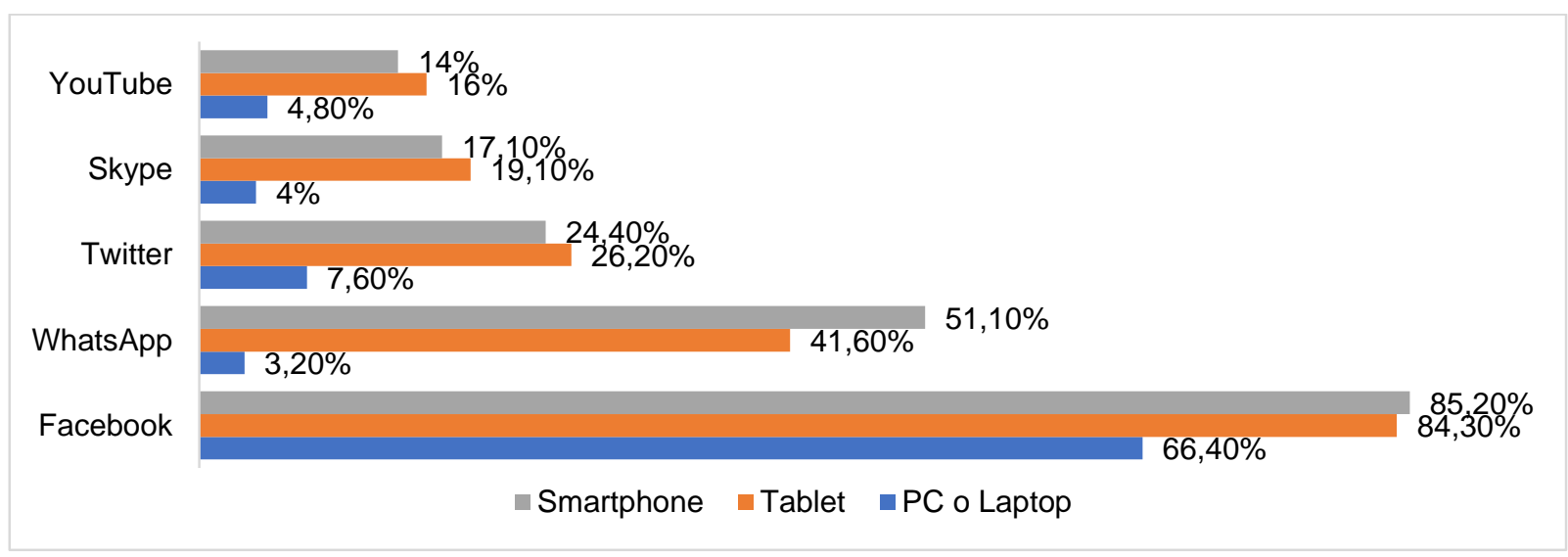

Fuente: Inec, 2015

Elaborado por: Los Autores

\section{MODELOS DE E-COMMERCE PARA LA REALIDAD ECUATORIANA}

A continuación se presenta un modelo de Canva para explicar como un modelo freemium en el e-commerce puede ser adaptado a la realidad ecuatoriana, puesto que con este modelo se cubren muchas necesidades de los usuarios ecuatorianos antes reflejadas como la inseguridad, costes, portabilidad, etc.; debido a que posee la comodidad de un uso gratuito en primera instancia brinda de primera mano la confianza que se necesita de la efectividad del producto y servicio y fomenta a que otros usuarios la recomienden aumentando así el reconocimiento de la marca.

Este término, popularizado en 2006 por Fred Wilson de Union Square Ventures, consiste en ofrecer de forma gratuita una parte básica del servicio o producto, con la opción de poder optar a un servicio avanzado con acceso a las características y funcionalidades avanzadas, previo pago de una tarifa o cuota mensual. El término "freemium" acuñado posteriormente por Jared Lukin es el resultado de la combinación de los dos aspectos que definen el modelo comercial: "free" y "premium". (Perez, 2013)

\section{Grafico 9. Modelo de Canva aplicado al modelo Freemium}

\begin{tabular}{|c|c|c|c|c|c|}
\hline \multirow{2}{*}{\begin{tabular}{|} 
Partners Clave \\
Diversos
\end{tabular}} & $\begin{array}{l}\text { Actividades Clave } \\
\text { Mejora } \\
\text { continua de la } \\
\text { plataforma }\end{array}$ & \multirow{2}{*}{\multicolumn{2}{|c|}{$\begin{array}{c}\text { Propuesta de valor } \\
\text { Producto o } \\
\text { servicio digital }\end{array}$}} & $\begin{array}{l}\text { Relación con los clientes } \\
\text { Atención simple y } \\
\text { Atención Premium }\end{array}$ & \multirow[b]{2}{*}{$\begin{array}{l}\text { Usuarios gratuitos } \\
\text { y usuarios pagos }\end{array}$} \\
\hline & $\begin{array}{l}\text { Recursos Clave } \\
\text { Plataforma } \\
\text { Digital }\end{array}$ & & & $\begin{array}{l}\begin{array}{l}\text { Canales de comunicación y } \\
\text { distribución }\end{array} \\
\text { Internet u otros canales } \\
\text { digitales }\end{array}$ & \\
\hline \multicolumn{3}{|c|}{$\begin{array}{l}\text { Estructura de costes } \\
\text { Costo de Mantenimiento de la plataforma }\end{array}$} & \multicolumn{3}{|c|}{ Gratuito y el valor unico o mensual } \\
\hline
\end{tabular}

Elaborado por: Los Autores 
Según (Perez, 2013) para que el modelo freemium funcione este debe ser capaz de subsistir con menos de un 10\% de usuarios de pago. Dicho de otro modo, un porcentaje de menos de un 10\% de usuarios de pago tiene que soportar a más de un $90 \%$ de usuarios gratuitos, y generar todavía beneficio. Pero existen muchas formas de plantear este modelo en función del servicio o producto a ofrecer.

A continuación, se presenta una tabla donde se diferencias ambos términos para poder tener una clara idea de lo que se va a tratar, la perspectiva que se trata de generar con esto es poder aprovechar las ventajas de ambos modelos para implementarlos a futuros emprendimientos ecuatorianos en las plataformas digitales.

Tabla 3. Aspectos fundamentales del modelo Dropshipping y el modelo Freemium

\begin{tabular}{|c|c|c|}
\hline Aspectos & Dropshipping & Freemium \\
\hline Características & $\begin{array}{l}\text { Compromiso del dropshipper: El } \\
\text { dropshipper no puede usar los datos del } \\
\text { cliente en su propio beneficio, tiene que } \\
\text { cumplir la cláusula de confidencialidad. } \\
\text { Esmerada atención al cliente: los } \\
\text { grandes mayoristas dropshipper suelen } \\
\text { tener comerciales con los que negociar, } \\
\text { de modo que la relación mercantil sea } \\
\text { más oficial y se puedan arañar } \\
\text { condiciones más ventajosas en la } \\
\text { negociación. (Rosado, 2015) }\end{array}$ & $\begin{array}{l}\text { Limitación por tiempo: Consiste en el } \\
\text { uso del producto por un periodo de } \\
\text { prueba, a partir del cual se bloquea } \\
\text { exigiendo el pago del producto para } \\
\text { volver a activarse. } \\
\text { Limitación por funcionalidad: } \\
\text { Consiste en limitar el acceso al grueso } \\
\text { de funcionalidades del servicio o } \\
\text { producto, con la posibilidad de acceder } \\
\text { a las características avanzadas, } \\
\text { funcionalidades y servicios adicionales } \\
\text { previo pago. } \\
\text { (Perez, 2013) }\end{array}$ \\
\hline Ventajas & $\begin{array}{l}\text { No existen preocupaciones } \\
\text { logísticas Es el proveedor el que se } \\
\text { ocupa de todos estos aspectos. } \\
\text { Formato de tienda online muy } \\
\text { escalable, por mucho que aumenten } \\
\text { las ventas, no van a aumentar los } \\
\text { gastos. (Rosado, 2015) }\end{array}$ & $\begin{array}{l}\text { Sin competencia: Una forma rápida de } \\
\text { eliminarnos posibles competidores es } \\
\text { ofrecer nuestro servicio a coste cero. } \\
\text { Feedback constante: Permite obtener } \\
\text { ir mejorándolo para adaptarse a las } \\
\text { demandas de los usuarios. } \\
\text { (ecaixapopular, 2015) }\end{array}$ \\
\hline Desventajas & $\begin{array}{l}\text { Importancia del tráfico web: si la } \\
\text { tienda dropshipping no destaca en la } \\
\text { web, no se cerrarán ventas por muy } \\
\text { completo que sea su catálogo o el } \\
\text { diseño de la página muy puntero. } \\
\text { Envíos múltiples: Para hacer un } \\
\text { pedido con artículos de dropshippers } \\
\text { diferentes, por lo que se enviarán } \\
\text { paquetes diferentes y esto puede hacer } \\
\text { que se asuman costes de envío } \\
\text { diferentes. } \\
\text { (Rosado, 2015) }\end{array}$ & $\begin{array}{l}\text { Alto coste: Sí, lo gratis vende, pero es } \\
\text { evidente que tiene un coste que } \\
\text { deberemos estar preparados para } \\
\text { afrontar. El retorno de la inversión en } \\
\text { estos casos se planifica a medio y largo } \\
\text { plazo. } \\
\text { Uso fraudulento: Las 'trampas' que los } \\
\text { usuarios utilizan para no pagar. Es } \\
\text { decir, un usuario se registra con } \\
\text { múltiples cuentas para disfrutar } \\
\text { siempre de la versión de prueba } \\
\text { premian gratuita. (ecaixapopular, 2015) }\end{array}$ \\
\hline
\end{tabular}

Elaborado por: Los Autores

Además de los modelos de comercio electrónico presentados, también se detallan los aspectos fundamentales del Webrooming y Showrooming, los cuales no solo se esperan puedan ser replicados en emprendimientos sino también 
en empresas ya consolidadas del mercado puesto que estos dos modelos permiten elaborar emprendimientos digitales, pero sin descuidar la parte física que es un factor que, en el país en muchos de los casos aumenta la seguridad y la confianza entre vendedor-comprador.

Factores irreplicables por tiendas online como la presencialidad y la inmediatez de la venta, poder ver y tocar los productos físicamente y la confianza que transmite la compra física libre de posibles estafas o fraudes. Además de ello, ha irrumpido con fuerza el concepto del 'Showrooming' y 'Webrooming', a través del cual los clientes planean sus compras online y las ejecutan en tiendas físicas o viceversa, beneficiándose de las diferentes ventajas que ello otorga. (Colino, 2015)

Tabla 4. Aspectos fundamentales del modelo Dropshipping y el modelo Freemium

\begin{tabular}{|c|c|c|}
\hline Aspectos & Webrooming & Showrooming \\
\hline Concepto & $\begin{array}{l}\text { Consiste en que el cliente potencial } \\
\text { haga el chequeo de sus productos de } \\
\text { interés en línea para luego realizar la } \\
\text { compra en la tienda física. } \\
\text { (Analiticaderetail, 2018) }\end{array}$ & $\begin{array}{l}\text { Se refiere a las compras en línea de } \\
\text { productos pero que solo se concretan } \\
\text { luego que el cliente las ha inspeccionado } \\
\text { en una tienda física. (Analiticaderetail, } \\
\text { 2018) }\end{array}$ \\
\hline \multirow[t]{3}{*}{ Características } & $\begin{array}{l}\text { No quieren gastar dinero extra en pago } \\
\text { de envíos. }\end{array}$ & $\begin{array}{l}\text { Mayor comodidad al poder comprar desde } \\
\text { sus dispositivos móviles. }\end{array}$ \\
\hline & $\begin{array}{l}\text { No quieren tomarse la molestia de } \\
\text { esperar varios días para recibir el } \\
\text { producto. }\end{array}$ & $\begin{array}{l}\text { Precios más bajos que en las tiendas } \\
\text { físicas. } \\
\text { Mayor variedad de marcas. }\end{array}$ \\
\hline & $\begin{array}{l}\text { Pueden regresar el producto a la tienda } \\
\text { de ser necesario. (Analiticaderetail, } \\
\text { 2018) }\end{array}$ & $\begin{array}{l}\text { Unir la experiencia virtual y la física. } \\
\text { (Analiticaderetail, 2018) }\end{array}$ \\
\hline
\end{tabular}

\section{Elaborado por: Los Autores}

Estos modelos de negocios de comercio electrónico presentados permiten al ecuador el poder minimizar en teoría los aspectos negativos detallados anteriormente según los estudios realizados en el país, dichos aspectos se han presentado en el país por lo cual no se ha podido potenciar dicho nicho de mercado con respecto a otros países, los presentamos como una perspectiva que el país puede tomar de referencia para futuros emprendimientos y no fracasar en el intento.

Cabe recalcar que no se asegura el éxito de uno u otro modelo y que la responsabilidad recae en el tipo de emprendedor y las decisiones que se lleven a cabo, pero consideramos que ante las negativas que se evidenciaron a través de los trabajos anteriores estas serían unas buenas opciones para poder tomar en cuenta al momento de plantearse la iniciativa de incursionar en este nuevo nicho de mercado.

\section{CONCLUSIÓN}

De lo antes expuesto en el desarrollo de este trabajo de investigación podemos concluir que el comercio electrónico es una gran opción por la que pueden optar los emprendedores del Ecuador, ya que en mucho de los casos, se requiere una inversión inicial mínima, y con un buen estudio de mercado y plan de marketing puede convertirse en un negocio escalable y mantener al margen a los competidores, todo es cuestión de la inversión que se efectué en el equipo creativo para poder llamar la atención de los potenciales clientes.

Se pudo evidenciar que muchos de los usuarios ecuatorianos no están familiarizados con los componentes tecnológicos o al menos existe un alto índice de desconfianza lo cual es un limitante a la hora de poder generarse la compra, por lo cual será necesario campañas de marketing mucho más inclusivas con el cliente. 
Muchos de los modelos de e-commerce antes mencionados pueden abrirse paso en el mercado ecuatoriano ofreciendo lo que el consumidor local requiere, pero se debe tener en cuenta las exigencias extranjeras al momento de llevar a cabo un modelo de negocio para una póstuma internacionalización.

\section{BIBLIOGRAFÍA}

1. Analiticaderetail. (12 de Septiembre de 2018). Obtenido de http://analiticaderetail.com/showroomingdiferencias-webrooming/

2. Colino, A. (2015). La irrupción del e-commerce y su influencia en la forma de vender de las empresas. Universidad Pontificia Comillas, Madrid. Obtenido de https://repositorio.comillas.edu/xmlui/bitstream/handle/11531/4560/TFG001264.pdf?sequence=1\&isAllowed=y

3. ecaixapopular. (2015). Obtenido de https://www.ecaixapopular.es/es/portal-emprendedoresvalencianos/recursos-herramientas-para-emprendedores/el-modelo-de-negocio-freemium-pros-y-contras/

4. Esparza, N. (2017). El Comercio Electrónico en el Ecuador. REVISTA CIENCIA E INVESTIGACIÓN, 29-32. Obtenido de https://revistas.utb.edu.ec/index.php/sr/article/view/119/pdf

5. Fernández, A., Sánchez, M., Jiménez, H., \& Hernández, R. (2015). La importancia de la Innovación en el Comercio Electrónico. Universia Business Review, 106-125. Obtenido de https://www.redalyc.org/pdf/433/43341001006.pdf

6. Korntheuer, R. (2016). SeoQuito. Obtenido de https://seoquito.com/comercio-electronico-en-ecuador/

7. Montalvo, J. (2016). Emprendimientos digitales y diseño de modelos de negocio: investigación aplicada en estudiantes de la Carrera de Comunicación. Contratexto, 155-170. Obtenido de http://revistas.ulima.edu.pe/index.php/contratexto/article/view/656/633

8. Ortiz, R., Camacho, F., \& Beltrán, R. (2017). Amazon vs. Walmart: ¿cuál de estas empresas dominará el comercio electrónico? REVISTA PUBLICANDO, 368-382. Obtenido de https://revistapublicando.org/revista/index.php/crv/article/view/500/pdf_324

9. Perez, M. (2013). blogthinkbig. Obtenido de https://blogthinkbig.com/ejemplos-de-exito-modelo-freemium

10. Pino, I., \& Salazar, X. (2018). Plan de marketing para el desarrollo de un canal de ecommerce para la empresa mercalimsa s.a. UNIVERSIDAD VICENTE ROCAFUERTE DE GUAYAQUIL, Guayaquil. Obtenido de http://repositorio.ulvr.edu.ec/bitstream/44000/2403/1/T-ULVR-2199.pdf

11. Ríos, A. (2015). ANÁLISIS Y PERSPECTIVAS DEL COMERCIO ELECTRÓNICO EN MÉXICO. Perfiles de las Ciencias Sociales, 61-87. Obtenido de http://revistas.ujat.mx/index.php/perfiles/article/view/993/848

12. Rosado, E. (2015). neoattack. Obtenido de https://neoattack.com/tienda-dropshipping-caracteristicas/

13. Sanabria, V., Torres, L., \& López, L. (2016). Comercio electrónico y nivel de ventas en las MiPyMEs del sector comercio, industria y servicios de Ibagué. Revista EAN, 241-250. Obtenido de http://www.scielo.org.co/scielo.php?script=sci_arttext\&pid=S0120-81602016000100010

14. Sanchez, J., \& Arroyo, F. (2016). Diferencias de la adopción del comercio electrónico entre países. Suma de Negocios, 141-150. Obtenido de https://www.sciencedirect.com/science/article/pii/S2215910X16300015

15. Sigmond, K. (2018). El comercio electrónico en los tratados de libre comercio de México. Revista IUS, 52-66. Obtenido de http://www.scielo.org.mx/scielo.php?script=sci_arttext\&pid=S1870-21472018000100359

16. Zamora, A. (2016). Disrupción digital: El efecto multiplicador de la economía digital. España: Accenture Strategy. Obtenido de https://circulodeempresarios.org/transformacion-digital/wpcontent/uploads/PublicacionesInteres/06.Accenture-Strategy-Digital-Disruption-Growth-Multiplier-Spanish.pdf 